\title{
Taste, odor, and tactile discrimination before and after smoking '
}

\author{
ROSE MARIE PANGBORN, IDA M. TRABUE ANO NINA BARYLKO-PIKIELNA2
}

UNIVERSITY OF CALIFORNIA, DAVIS

Differential sensitivity was measured before and after smoking a cigarette by seven smokers, using six non-smokers as controls. Stimuli consisted of the tastes of sucrose, sodium chloride, citric acid, caffeine, and quinine hydrochloride; the odors of vanillin and 2-butanone; and the viscosity produced by a carrageenan gum. No significant jinds were observed between smokers and non-smokers, nor before vs after smoking. Therefore, the data supported neither longterm nor short-term influences of cigarette smoking. Practice effects were noted among the control group, as more correct responses were obtained on the second than on the first set of solutions in most of the test stimuli.

Popular opinion contends that smoking decreases taste sensitivity, reduces olfactory perception, diminishes hunger contractions, and interferes with the recognition of oral chemical stimuli and the appreciation of fine foods. Valid experimental evidence supports only a few of these claims, with considerable lack of agreement among investigators. Although Arfmann and Chapanis (1962) compared only three smokers and three nonsmokers and used a questionable procedure of spraying the nostrils with vanilla extract which is high in alcohol, they noted that smokers consistently rated intensities lower than nonsmokers. These workers mentioned the observations of Sinnot and Rauth (1937), and Hall and Blakeslee (1945), that absolute taste thresholds were higher among smokers than among nonsmokers and advance two hypotheses in explanation: (1) Nicotine and rutin in tobacco inhibit neural activity in the tongue and nasal passages; and (2) Combustion products from tobacco form a layer inside the mouth and nose preventing stimuli from coming into contact with the receptor sites. Relative to the first hypothesis, early work by Zotterman (1944) indicated that when nicotine in doses from $0.005-0.5 \mathrm{mg}$ was introduced into the lingual artery of the cat via a cannula inserted into the thyroid artery, neither the tactile fibers terminating in the tongue nor the taste fibers showed traces of an augmented activity. The inhibition of neural activity could occur after long term usage of tobacco, whereas the formation of a film or layer in the oral and nasal cavity could possibly result from single exposure to combustion products.

Generally, both smokers and nonsmokers have been found equally useful for psychophysical studies on chemoreception and food evaluation (Amerine et al, 1965). Ss are usually advised to refrain from smoking for $30-60$ min prior to a test session, mainly so that they do not introduce tobacco odors into the test room where it could distract nonsmokers. However, no experimental data are available on the short term or immediate effects of smoking on oral sensory perception.

The investigations described herein were undertaken to measure differential sensitivity among suprathreshold concentrations of taste, odor, and oral tactile stimuli, before and after smoking of the first cigarette of the day, using nonsmokers as controls to check for practice effects.

\section{Subjects}

\section{METHOD}

Seven cigarette smokers and six nonsmokers were selected on the basis of their taste and olfactory sensitivity, their interest in participating, and their availability throughout a five month period. Five of the smokers used less, and two used more than one pack of cigarettes per day. The Ss were departmental employees, seven females and six males, approximately the same number within each group. They ranged in age from 23 to 48 years.

\section{Test Stimuli}

The following compounds, of reagent grade purity, were used as stimuli:

Taste-sucrose, sodium chloride, citric acid, caffeine, and quinine hydrochloride;

Odor-vanillin and 2-butanone;

Tactile-carrageenan gum.

The taste compounds were selected to represent simple sweet, salty, sour, and bitter sensations. Two bitter compounds were included to check the observations of Krut et al (1961) and Kaplan et al (1964) that taste thresholds for bitterness were significantly higher in smokers than in nonsmokers, whereas sweet, sour, or salty thresholds showed no differences. Vanillin and 2-butanone were used because of their pleasant, food-like odor qualities (vanilla and bleu cheese, respectively), and their stability and solubility in water. The food grade carrageenan gum (Genulacta $\mathrm{K} 100^{\circ}$, supplied by the Uni-Gum Division of T. M. Duche \& Sons, Inc., Clifton, N. J.) effectively increased the viscosity of the solutions without imparting odors or tastes. Low red illumination eliminated the slight visual differences caused by light reflectance from the surface. 


\section{Test Procedure}

Differential sensitivity was measured by a method of constant stimuli, described in detail by Galanter (1962). A standard suprathreshold concentration of moderate intensity was presented with a comparison stimulus of either lower or higher concentration. Eight comparison stimuli, equidistant in concentration, and designated as $-4,-3,-2,-1,+1,+2,+3$, and +4 , were evaluated against the standard. Concentrations of the comparison stimuli were selected by prior screening to yield an overall correct response of approximately $75 \%$. Pairs within a set of eight and samples within a pair were presented in randomized, counterbalanced order. Taste and tactile stimuli were served in aliquots of $30 \mathrm{ml}$ in $50 \mathrm{ml}$ beakers containing three digit code numbers. Vanillin and 2-butanone were dispersed in distilled water within $1 / 2 \mathrm{~h}$ prior to serving. Samples of $10 \mathrm{ml}$ were placed in stemmed wine glasses and covered with aluminum caps containing three digit code numbers. Ss handled the glasses by the stem to avoid heating the solutions with their hands, swirled the contents gently, removed the cap, and took three quick sniffs from the headspace above the liquid. Ss were requested to circle the code number of the sample with the greater intensity within each pair. For taste and tactile stimuli, distilled water was used for oral rinsing prior to testing of each pair. Cuspidors were furnished for expectoration as swallowing was not permitted. All solutions were served at room temperature $\left(22^{\circ} \mathrm{C}\right)$. For each test, Ss were seated comfortably in individual, partitioned booths, separated from the preparation area. To stimulate interest, Ss were informed of their results immediately after each test session. Tests were conducted between 8:30 and 9:30 AM, Monday through Friday, with four to seven test sessions for each of the eight compounds. The first session for each compound was considered a practice period and these results were not included in the final data.

\section{Smoking Procedure}

Smokers were assigned a specific time of testing and requested to refrain from smoking since the previous night. First the $\mathrm{S}$ evaluated a set of eight pairs, then was given a cigarette and instructed to smoke $45 \mathrm{~mm}$ of it in another room. Since only two Ss inhaled normally, all seven were instructed not to inhale during the smoking of the test cigarette. Immediately after smoking the cigarette, the $S$ washed his hands, returned to the test room, and received a second set of eight pairs for evaluation. The only difference between the first and second sets was order of presentation among and between pairs.

The nonsmokers served as controls, receiving the first set of samples, followed by a 5 min rest, then testing the second set of samples.

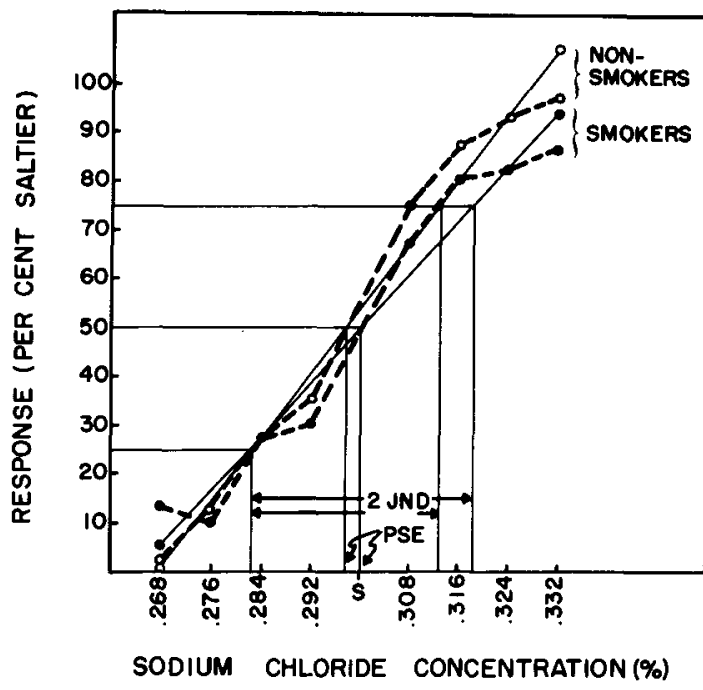

Fig. 1. Response of smokers and non-smokers to sodium chloride. The standard concentration (S), against which the four lower and the four higher levels were compared, is . $30 \%$ sodium chloride. The broken lines represent observed responses with a solid regression line plotted for each group. The points of subjective equality (PSE) are calculated by bisecting the regression lines at $50 \%$ response. One just noticeable difference (JND) is one-half the distance between $25 \%$ and $\mathbf{7 5} \%$ response. For sodium chloride, the jnds for smokers and non-smokers and .021\% and .015\% respectively.

\section{Analy sis of Data}

For calculation and plotting, the data were subdivided into three groups:

(1) Responses of seven smokers . vs six nonsmokers to both sets of samples

(2) Responses of seven smokers to Set 1 evaluated immediately before smoking a cigarette vs Set 2 evaluated immediately after smoking a cigarette

(3) Responses of six nonsmokers to Set 1 vs Set 2 to determine any practice effects.

Correct responses to each pair were plotted as the percentage ascribing greater sensory intensity against the concentration of the compound, as shown in Fig. 1. A regression line was calculated to fit each set of data and the just noticeable difference (jnd) determined as half the distance between $25 \%$ and $75 \%$ correct response. The $50 \%$ point, i.e., the point of subjective equality (pse) was also recorded from the graph. Student's $t$ test for nonpaired variates was applied to determine significant differences within the three categories outlined above. Data from each of the eight compounds are presented in terms of percent correct response, jnd, and pse, with the latter two values expressed in terms of absolute concentration (\%) as well as in concentration units so that cross comparisons could be made among compounds.

\section{RESULTS AND DISCUSSION}

Tables 1 and 2 summarize responses to the eight 
Table 1. Correct response, just noticeable differences, and points of subjective equality to taste stimuli among smokers and non-smokers.

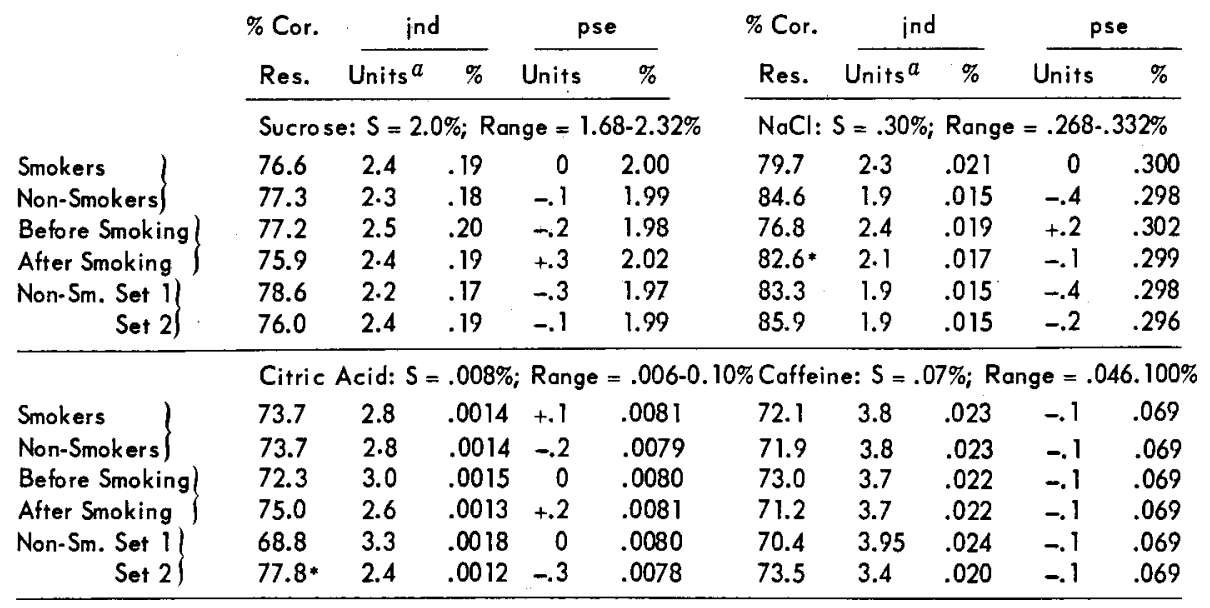

a Units $=.08 \%$ for sucrose: $.008 \%$ for $\mathrm{NaCl}$; $.0005 \%$ for citric acid: $.006 \%$ for caffeine.

* Significant difference in correct response at $p<0.05$.

sensory stimuli among and between smokers and the nonsmoking controls. Ease of reproducibility of judgments determined the number of test days spent on each compound, so that the total number of individual responses constituting the percentages for smokers and nonsmokers in the two tables are:

$\begin{array}{cc}\text { Smokers } & \text { Nonsmokers } \\ \mathbf{n} & \mathbf{n} \\ 448 & 384 \\ 448 & 384 \\ 560 & 480 \\ 576 & 576 \\ 672 & \mathbf{5 7 6} \\ 672 & 576 \\ 756 & 648 \\ 784 & 672\end{array}$

No significant differences in sensitivity were observed between the smokers and nonsmokers, although slightly smaller jnds were obtained for the latter group in five out of eight stimull.

Sensitivity before vs after smoking resulted in only one significant difference, but in an unanticipated direction, i.e., $82.6 \%$ correct response to sodium chloride after, compared to $76.8 \%$ before smoking. Practice effects may have been operative since, in six of the eight stimuli, slightly lower jnds were obtained after smoking the cigarette. Among the nonsmokers, the jnds were lower on the second trial for caffeine, quinine, 2-butanone, and citric acid, significantly so for the latter. These four stimuli have more of a lingering aftereffect than do sucrose, sodium chloride, vanillin, and the gum.

Table 2. Correct response, just noticeable differences, and points of subjective equality to taste, odor, and tactile stimuli among smokers and non-smokers.

\begin{tabular}{|c|c|c|c|c|c|c|c|c|c|c|}
\hline & \multirow{2}{*}{$\begin{array}{l}\% \text { Cor. } \\
\text { Res. }\end{array}$} & \multicolumn{2}{|c|}{ ind } & \multicolumn{2}{|c|}{ pse } & \multirow{2}{*}{$\begin{array}{l}\text { \% Cor. } \\
\text { Res. }\end{array}$} & \multicolumn{2}{|c|}{ jnd } & \multicolumn{2}{|c|}{ pse } \\
\hline & & Units $a$ & $\%$ & Units & $\%$ & & Units $a$ & $\%$ & Units & $\%$ \\
\hline & \multicolumn{10}{|c|}{ Quinine: $S=.0025 \%$; Range $=.0017-.0033 \%$ Vanillin: $S=.003 \% ;$ Range $=.00$ 14-.0046\% } \\
\hline Smokers $\}$ & 74.7 & 2.9 & .00057 & +.1 & .00252 & 61.4 & 4.7 & .0019 & -.3 & .00288 \\
\hline Non-Smokers] & 71.5 & 2.9 & .00057 & +.1 & .00252 & 68.5 & 3.0 & .0012 & +.2 & .00308 \\
\hline Before Smoking & 76.4 & 2.7 & .00054 & +.1 & .00252 & 61.4 & 4.8 & .0020 & -.1 & .00296 \\
\hline After Smoking $\}$ & 72.9 & 3.2 & .00064 & +.2 & .00254 & 61.4 & 4.7 & .0019 & -.5 & .00280 \\
\hline \multirow{3}{*}{$\left.\begin{array}{r}\text { Non-Sm. Set } 1 \\
\text { Set } 2\end{array}\right\}$} & 68.1 & 3.2 & .00064 & +.3 & .00256 & 69.6 & 2.8 & .0011 & +.3 & .00304 \\
\hline & 75.0 & 2.5 & .00050 & 0 & .00250 & 67.5 & 3.1 & .0012 & +.1 & .00312 \\
\hline & \multicolumn{3}{|c|}{ 2-Butanone: $\mathrm{S}=.0032 \%$; } & Range= & $=.0016-$ & \multicolumn{5}{|c|}{ Gum: $S=.16 \% ;$ Range $=.08-.24 \%$} \\
\hline Smokers & 63.4 & 3.95 & .00158 & +.6 & .00344 & 69.8 & 3.2 & .064 & -.3 & .154 \\
\hline Non-Smokers\} & 72.2 & 2.9 & .00114 & +.7 & .00348 & 70.1 & 3.2 & .063 & -.3 & .154 \\
\hline Before Smoking) & 61.5 & 4.3 & .00192 & +.9 & .00356 & 67.6 & 3.9 & .076 & -.5 & .150 \\
\hline After Smoking \} & 65.3 & 3.4 & .00136 & +.4 & .00336 & 72.0 & 2.8 & .056 & -.2 & .156 \\
\hline Non-Sm. Set 1$\}$ & 69.0 & 3.2 & .00128 & +.7 & .00348 & 71.5 & 2.9 & .058 & -.6 & .148 \\
\hline Set 21 & 75.3 & 2.6 & .00104 & +.6 & .00344 & 68.8 & 3.4 & .068 & +.1 & .162 \\
\hline
\end{tabular}

a Units $=.0002 \%$ for quinine; $.0004 \%$ for vanillin; $.0004 \%$ for 2 -butanone; $.02 \%$ for gum. 
Inspection of the data for vanillin revealed that we fell slightly below the desired overall correct response of $75 \%$. We were prevented from repeating this series using a wider range of concentrations owing to the unavallability of two smokers for further testing.

Expressing the jnd and pse values in the form of a common unit facilitated within- and among-stimuli comparisons; however, it is not possible to say that among-stimuli jnds are subjectively equal, as the range of concentrations employed influenced the slope of the regression line. Furthermore, it should be pointed out that the psychometric function being measured is sigmoid but, within the range of concentrations tested, the observed responses could be approximated reliably by a straight line.

Values for the pse are included for comparison, as it is well known that the difference between the point of subjective equality and the point of objective equality becomes smaller as discrimination improves. Referring to loudness as a stimulus example, Galanter (1962) stated that the pse usually falls at a stimulus point less than that of the standard stimulus, attributable to a "time order error." In the data reported herein, negative pse values were obtained for caffeine and the gum, positive values for 2-butanone and quinine, with mixed values for the remaining stimull.

Although it can be concluded that among the small group of Ss and stimuli tested, smoking did not influence chemical or tactile sensitivity, within-s response to long term changes in smoking patterns remains to be established.

\section{References}

Amerine, M. A., Pangbom, R. M., \& Roessler, E. B. Principles of sensory evaluation of food. New York: Academic Press, 1965. P. 292.

Arfmann, B. L., \& Chapanis, N. P. The relative sensitivities of taste and smell in smokers and non-smokers. J. gen. Psychol., 1962, 66, 315-320.

Galanter, E. Contemporary psychophysics. In New directions in psychology. New York: Holt, Rinehart \& Winston, 1962. Pp. 87156.

Hall, A. R., \& Blakeslee, A. F. Effects of smoking on taste thresholds for phenyl-thio-carbamide (PTC). Proc. nat. Acad. Sci., $1945,31,390-396$.

Kaplan, A. R., Glanville, E. V., \& Fischer, R. Taste thresholds for bitterness and cigarette smoking. Nature, 1964, 202, 1366.

Krut, L. H., Perrin, M. J., \& Bronte-Stewart, B. Taste perception in smokers and non-smokers. Brit. Med. J., 1961, 1, 384-387.

Sinnot, J. J., \& Rauth, J. E. Effect of smoking on taste thresholds. J. gen. Psychol., 1937, 17, 151-153.

Zotterman, Y. A note on the action of lobeline, nicotine and acetylcholine on the afferent nerves of the tongue. Acta Physiol. Scand., 1944, 8, 377-379.

\section{Notes}

1. Supported in part by the Council for Tobacco Research-U.S.A.

2. Dr. Pikielna is on leave of absence, $1967-1968$, from the Polish Meat Research Institute, Warsaw.

(Accepted for publication July 14, 1967.) 